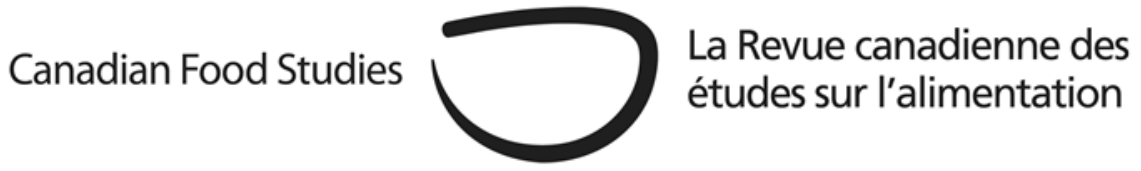

Original Research Article

\title{
Examining the relationship between food security and perceived health among Memorial University students
}

\author{
Lisa Blundella* and Maria Mathews ${ }^{b}$ \\ ${ }^{a}$ Memorial University of Newfoundland \\ ${ }^{b}$ Western University
}

Abstract

Objectives: The prevalence of student food insecurity at Memorial University of Newfoundland (MUN) is relatively high (58.0\%) compared to the national population (12.7\%). We explored the relationship between food security status and perceived health, as well as the qualitative nature of student experience due to food insecurity among MUN students.

Methods: Through an online survey of returning MUN students at the St. John's campus, we assessed food security using Statistics Canada's Canadian Household Food Security Survey Module (HFSSM), and self-reported physical health, mental health, and stress. We used logistic regression to compare health and stress ratings between students of different food security levels. We thematically coded open-ended responses to describe students' experiences related to food insecurity.

Results: Among the 967 study eligible students, $58.01 \%$ were considered food insecure, of which $18.10 \%$ were marginally food insecure, $28.23 \%$ were moderately food insecure, and $11.69 \%$ were severely food insecure. After controlling for significant predictors, students who were moderately or severely food insecure were 2.09 [95\% CI:(1.39, 3.13)] and 3.41 [95\% CI:(2.09, 5.55)] times as likely to rate their physical health as 'fair' or 'poor' than food secure students, and 2.17 [95\% CI:( $(1.54,3.05)]$ and 5.54 [95\% CI:(3.47, 8.85)] times as likely to rate their mental health as 'fair' or 'poor' than food secure students, respectively. 
Moderately food insecure and severely food insecure students were 1.67 [95\% CI:(1.22, 2.29)] and 2.21 [95\% CI:(1.42, 3.43)] times as likely to rate their stress level as 'high' than food secure students, respectively. Students' comments on their experience were categorized into four themes: Impact on academic performance, impact on student experience, impact on physical health, and impact on mental health and/or stress.

Conclusion: Food security levels experienced by MUN students was closely related to their perceived physical and mental health. As food security levels worsened among participants, their self-reported physical and mental health also worsened. Health professionals working with university student populations should screen for food security and consider its relationship to students' health.

Keywords: Food supply; food insecurity; hunger; student health services; universities; population health

\section{Introduction}

Food insecurity exists when an individual has insufficient access to food due to financial constraints (Tarasuk et al., 2014). The experience of food insecurity has long been linked with numerous health concerns, such as an inadequate diet, chronic diseases, and depression (Kirkpatrick \& Tarasuk, 2008; Vozoris \& Tarasuk, 2003). Researchers are continually learning more about food security's relationship with health, and recent findings corroborate that food insecurity is associated with suicidal ideation, increased utilization of mental health care services, and increased health care utilization costs (Davison et al., 2015; Tarasuk et al., 2015; Tarasuk et al., 2018).

Food security research among the university population has been gaining traction in recent years, with student poverty recognized as a growing problem in light of decreased public funding for universities, increased tuition rates and student fees, and stagnant student funding (Canada Without Poverty, 2017; Smith-Carrier, 2020). Within Canada, researchers have found rates of campus food insecurity (moderate and severe levels) between $28.6 \%$ and $46 \%$ across the country (Entz et al., 2017; Olauson et al., 2018; Silverthorn, 2016), which are alarmingly higher than the national household food insecurity prevalence of $8.7 \%$ (moderate and severe levels) reported for Canada in 2018 (Statistics Canada, 2020). The health effects of experiencing food insecurity during postsecondary education are not well known, but preliminary research suggests that food insecurity among postsecondary students is associated with high stress, depression, and self-reported poor physical and mental health (Farahbakhsh et al., 2017; Frank, 2018; Hughes et al., 2011; Patton-López et al., 2014). 
Evidence also strongly suggests that the burden of hunger and stress associated with experiencing food insecurity may cause the inability to concentrate and compromise academic success (Bessey et al., 2020; Farahbakhsh et al., 2017; Maroto et al., 2015; Patton-López et al., 2014).

The objective of our study was to determine if food security level was a significant predictor of perceived physical health, perceived mental health, and/or stress level among students at Memorial University of Newfoundland (MUN). An estimated 58.0\% of MUN students experienced some level of food insecurity (marginal, moderate, or severe) in the 20152016 academic year (Blundell et al., 2019). We hypothesized that experiencing food insecurity is associated with poorer perceived physical health, poorer perceived mental health, and increased stress among the MUN student population. Our study provides a better understanding of the factors related to food insecurity among postsecondary students and provides university administration and food security policy actors to form evidence-based recommendations to improve the well-being of students.

\section{Methods}

In Fall 2016, we disseminated a cross-sectional online survey to returning MUN students. We pre-tested our survey instrument to identify any potential errors or confusing survey questions. Nine university alumni of various backgrounds pre-tested the survey and we amended the instrument to improve clarity, but did not make any changes to the questions related to perceived health or food security status.

Inclusion and exclusion criteria were set to ensure participants shared a similar food environment. Participants were included if they had been enrolled in full- or part-time studies at a MUN campus in St. John's, Newfoundland and Labrador (NL), including the St. John's campus, the Marine Institute, and the Centre for Nursing Studies, during the September 2015 to April 2016 academic year. Our survey questions referred to the previous academic year, and therefore, we excluded students who were currently enrolled in their first year of studies. Postgraduate medical residents were also excluded. Based on our criteria, 10,400 of 18,272 students attending MUN in 2015 were eligible for our survey that was conducted in 2016.

\section{Recruitment}

We recruited students by distributing posters on St. John's campuses, sharing advertising posts on social media pages related to MUN students such as student societies' Facebook and Twitter pages, and sending emails to the email lists of MUN graduate, undergraduate, and international student associations and the international advising office. 
Each academic unit at St. John's campuses was also contacted and asked to email our survey to their corresponding list of students. Participating student groups and academic units were then asked to email students at the beginning of the study, and again one week before the study ended. The survey link was live from mid-September to mid-October 2016, for four weeks total. As an incentive to participate in the study, we offered students a chance to win one of five $\$ 50$ grocery gift cards. This incentive was advertised in each recruitment communication, and students were informed that their survey answers were anonymous and not tied to contact information provided for the prize draw.

\section{Assessing food security and health status}

The Household Food Security Survey Module (HFSSM) is used in the Canadian Community Health Survey (CCHS) to evaluate household food security (Health Canada, 2008). The HFSSM contains eighteen questions relating to food security during the past twelve months, ten of which form the 'Adult Scale', specific to the experience of the adult(s) of the household. This module is in a standardized survey instrument used in the CCHS. For this study, we used the Adult Scale with minor wording modifications to change second person statements (you) to first person (I) statements. For example, the HFSSM question "You and other household members worried food would run out before you got money to buy more", was changed to "I worried whether my food would run out before (I/we) got money to buy more.” These changes were made to remain consistent with other food security research recently conducted in postsecondary student populations (Frank, 2018; Silverthorn, 2016). These questions referred to students' food experience during the previous 12 months. For this reason, students were asked to relate all questions about their demographics and experience to the last academic year, and this was repeatedly noted across the survey. Students were also asked to only include people who they shared groceries with regularly when selecting responses. Consistent with an interdisciplinary research team investigating household food insecurity in Canada, PROOF, we classified students as 'food secure' if they had zero affirmative responses, 'marginally food insecure' if they had one affirmative response, 'moderately food insecure' if they had two to five affirmative responses, and 'severely food insecure' if they had five or more affirmative responses (Tarasuk et al., 2014). Health Canada (2008) has traditionally not recognized 'marginal food insecurity' as a category, and instead has considered these individuals as 'food secure', but otherwise has scored food insecurity in the same way.

We used a five-point Likert scale to assess participants' perceived physical health and mental health during the past twelve months, with options of 'poor', 'fair', 'good', 'very good', and 'excellent'. Because answers were highly skewed, we re-aggregated physical and mental health ratings into two categories. 'Poor' and 'fair' ratings were grouped as 'poor' perceived health, and 'good', 'very good', and 'excellent' were grouped as 'good' perceived health. 
We also assessed participants' level of stress using a five-point Likert scale, asking student to describe whether most days in the last twelve months were 'not at all stressful', 'not very stressful', 'a bit stressful', 'quite a bit stressful', or 'extremely stressful'. Responses of 'quite a bit stressful' and 'extremely stressful' were grouped as high stress, and 'not at all stressful', 'not very stressful', and 'a bit stressful' were grouped as low stress.

We collected demographic information such as student origin, level of study, gender, age, course load, partner status, parental status, living situation, and primary source of income. We also included an open-ended question asking participants to identify how the quality of their university experience has been negatively affected by a lack of money for food.

\section{Analysis}

Food security level was the independent variable in this study, measured using the HFSSM. Covariates included sociodemographic (gender, marital status, parental status, Indigenous status), educational (student's level of study, year of study, course load, year in program), financial (primary income source, employment status), food-related characteristics (meal plan, grocery sharing), and living arrangements. These variables were chosen based on reviewing literature related to postsecondary student food security and a discussion of factors that are unique to the student food experience (i.e., meal plan, students having roommates which may or may not practice grocery sharing). Our dependent variables were the students' perceived physical health, mental health, and stress level.

We used SPSS (Statistical Package for the Social Science-v22) to describe the characteristics of the sample. We used Chi-square tests (or Fisher's exact test in cases of small sample sizes) to assess each outcome and predictor variable, and then used multiple logistic regression to investigate how food security level related to health and stress, after controlling for other significant predictors. Covariates that were significant in the bivariate analyses were included in regression models (data not shown). This included the following covariates: Student's level of study, place of origin, gender, parent status, year of study, and primary source of income. Covariates were removed from the model if they were not significant (using the Wald test) and if they did not significantly improve the change in the -2 log likelihood value (Tabachnick \& Fidell, 2007). Only significant predictors were included in the final regression models.

We thematically coded responses to open-ended questions to capture students' perceptions of the effect of food insecurity on physical health and well-being, and academic performance. The questions asked, "Do you have any comments on how a lack of money for food has affected the quality of your university experience?" and at the end of the survey, "Do you have any further comments?" Two authors reviewed all responses independently and developed a coding template with preliminary themes (Fink, 2013). 
Both authors then met to compare themes, refine the meaning of each theme, and then recoded the data using the final coding template. We both independently reviewed the coding of quotations and together we resolved any discrepancies. We present illustrative quotations for each theme.

\section{Results}

Our survey had a total of 1486 participants. Of these, we excluded 326 who were not returning MUN students, 148 who did not complete the required screening questions for food insecurity status and health status, and 45 who attended a campus other than St. John's campus, for an eligible sample of 967 (Figure 1). The sample was representative of the total student population in terms of student origin $(66 \% \mathrm{NL}, 19 \%$ Out-of-province [OOP] Canadian, 13\% international in the population; $65.42 \% \mathrm{NL}, 20.29 \%$ OOP, $14.29 \%$ international in the sample; $\chi 2=2.12, \mathrm{p}=$ $0.3465)$ and level of study (21.6\% graduate and $78.4 \%$ undergraduate in the population; $22.49 \%$ graduate and $73.26 \%$ undergraduate in the sample; $\chi 2=1.83, p=0.1761$ ).

The majority of respondents in the study sample were undergraduate students $(73.26 \%)$, from NL (65.42\%), full-time students (92.95\%), female (73.25\%), and living off-campus $(85.52 \%)$ (Table 1). We did not have sample frame data to assess representativeness using each of these characteristics. However, Statistics Canada (2016) reported that $54 \%$ of college and university graduates of NL were women in 2013. This indicates that women may be overrepresented in our sample. Less than half (41.99\%) were food secure and more than one in ten students (11.69\%) were severely food insecure. The proportion of students (by food security level) who reported poor physical health, poor mental health, and high stress were significantly different from expected values, suggesting that food security status is related to health and stress outcomes (Table 2).

One fifth of the students (19.96\%) rated their physical health as poor. After controlling for other significant predictors graduate students were 1.89 times less likely (the inverse of 0.53 ) to report poor physical health than general undergraduate students. Compared to food secure students, marginally, moderately, and severely food insecure students were 1.74, 2.09, and 3.41 times more likely, respectively, to report poor physical health (Table 3).

More than a third of the students (38.37\%) reported poor mental health. After controlling for other significant predictors, graduate students and medical students were 1.79 and 2.5 times less likely respectively (the inverse of 0.56 and 0.40 ), to report poor mental health than other undergraduate students.

Female students were 2.73 times more likely to report poor mental health than male students. Marginally, moderately, and severely food insecure students were 2.25, 2.17, and 5.54 times more likely, respectively, to report poor mental health than food secure students (Table 3 ). 
More than half the students $(54.60 \%)$ reported high stress. Female students were 1.75 times more likely than male students to report high stress. After controlling for gender, marginally, moderately, and severely food insecure students were 1.27, 1.67, and 2.21 times more likely, respectively, to report high stress (Table 3).

From students' open-ended responses on how food insecurity has impacted their university experience, we identified recurring themes related to the impact on physical health, impact on mental health and stress, impact on academic performance and impact on student experience (Table 4). Comments related to "impact on physical health" were focused on feelings of low energy and specific health implications such as anemia and hypertension. One student indicated that they "suffer from constant tiredness and weakness" (Participant 109800994), while another stated that "[worry about food supply] ...contributed to [their] sickness and deferred exams." (Participant 109525180) In the theme of "impact on mental health/stress", students discussed their stress and anxiety associated with acquiring a sufficient food supply. The theme "impact on academic performance" relates to how food insecurity affects a student's experience in the classroom, studying, ability to focus, and succeeding in their program of study. Several participants of our study stated that they found it difficult to maintain attention, energy, and focus when experiencing hunger or a compromised food intake. Students also commented on how food security shaped their overall experiences as university students. While attending postsecondary training is often described as a period when young adults form many important lifelong relationships through social interactions, food insecure students commented that they were often unable to socialize due to having to save money or to work for money to buy food.

\section{Discussion}

Food insecurity is associated with poorer self-reported physical and mental health and higher levels of stress among university students enrolled at MUN, NL's sole university. The findings are consistent with other studies of postsecondary student populations across North America and the general Canadian adult population (Frank, 2018; Hughes et al., 2011; Patton-López et al., 2014; Vozoris \& Tarasuk, 2003). Moreover, in this study, there is a direct relationship between the level of food security and health, as food insecurity worsened, self-reported physical health, mental health, and stress worsened among participants. In addition, as shown through responses to open-ended questions, food insecurity also influences academic performance and student experiences. These findings highlight the need for greater public health interventions, from the university as well as other organizations, to address the high prevalence of food insecurity among postsecondary students. For example, MUN students who rely on student grants and loans are vulnerable to food insecurity, and policies that include changes to government funding available to students may increase students' ability to pay for food (Blundell et al., 2019). 
Other vulnerable student populations at MUN include students living off-campus and students with children (Blundell et al., 2019). Affordable housing and childcare policies may alleviate the financial pressure experienced by these groups. Also, despite available programs at MUN, such as food banks on campus, few students are aware of or use these programs (Blundell \& Mathews, 2021). The food insecurity experiences of our study participants emphasize the need to tailor programs to the needs of different student populations. International students are vulnerable to food insecurity and are more likely to use MUN campus resources to access food (Blundell \& Mathews, 2021). MUN should conduct a further program evaluation of these services and collect feedback from international students to learn how to better support these individuals.

Additionally, because international students are often not eligible to receive funding from the same opportunities as other students, an increase in funding options available to these students (offered by MUN or other institutions) may prevent the financial strain that leads to food insecurity in this population.

Stress and mental health impacts of food insecurity were gendered, while physical health was not. These findings are consistent with studies that show women are more likely to report mental health-related issues than men (Nurullah, 2010). Moreover, while women typically report poorer perceived physical health than men, university students are generally a young and relatively physically healthy population. Furthermore, many students may experience food insecurity for the first time if they have moved away from home and/or assumed responsibility for paying for food for the first time. The cumulative effects of food insecurity after a relatively short exposure may be limited for an otherwise healthy population, which may have contributed to students less frequently reporting poor physical health at this point in time. Future studies that involve following a cohort of postsecondary students over time would help us better understand the relationship between food insecurity and health in this population.

Graduate students and medical students who were food insecure were generally less likely to report poorer physical and mental health. However, given that graduate studies and medicine admit high performing students, and students from wealthier families are more likely to pursue graduate studies and medical careers (Dhalla et al., 2002), these findings may reflect a selection bias. This bias means that food insecure graduate students may have better overall health, and subsequently better perceived health, given that more graduate students come from wealthier families as compared to undergraduate students. While students' comments indicate that food insecurity may also affect academic performance, further research is needed to assess how food insecurity affects career goals and trajectories.

This study has limitations. For instance, because this is a cross-sectional study, the direction of causality between health and food insecurity cannot be determined.

One might suggest that the arrow has the potential to be bi-directional; food insecurity can cause malnutrition and stress (decreased health), and the presence of chronic health conditions may increase vulnerability to food insecurity by affecting one's income and ability to access food. 
Our study also used non-random sampling and is subject to sampling bias. Women are likely overrepresented in this study. The use of self-reported health measures also presents caution for interpretation. Further, while we slightly rephrased statements for our context, the HFSSM is intended to be used at a household level, rather than an individual level. To build on existing knowledge, future researchers of student food insecurity should use methods that enable us to infer causation so that we can better analyze food insecurity's relationship with health and include objective (non-survey) data sources.

\section{Conclusion}

Food security is directly associated with physical health, mental health, and overall stress levels of university students in NL. Food insecurity may also influence academic performance and contribute to systemic inequities in professional programs. Given the high prevalence of food insecurity among postsecondary students, food insecurity presents an important and urgent public health challenge across Canada.

\section{References}

Bessey, M., Frank, L., \& Williams, P.L. (2020). Starving to be a student: The experiences of food insecurity among undergraduate students in Nova Scotia, Canada. Canadian Food Studies, 7(1), 107-125. https://doi.org/10.15353/cfs-rcea.v7i1.375.

Blundell, L., \& Mathews, M. (2021). Identifying coping strategies among food insecure university students. Manuscript in preparation.

Blundell, L., Mathews, M., Bowley, C., \& Roebothan, B. (2019). Determining student food insecurity at Memorial University of Newfoundland. Canadian Journal of Dietetic Practice and Research, 80(1), 14-21. DOI: 10.3148/cjdpr-2018-026

Canada Without Poverty. (2017). The cycle of student debt and poverty-and how we aren't ending it. https://cwp-csp.ca/2017/09/the-cycle-of-student-debt-and-poverty-and-howwe-arent-ending-it.

Davison, K.M., Marshall-Fabien, G.L., \& Tecson, A. (2015). Association of moderate and severe food insecurity with suicidal ideation in adults: National survey data from three Canadian provinces. Social Psychiatry and Psychiatric Epidemiology, 50(6), 963-972. DOI: 10.1007/s00127-015-1018-1 
Dhalla, I.A., Kwong, J.C., Streiner, D.L., Baddour, R.E., Waddell, A.E., \& Johnson, I.L. (2002). Characteristics of first-year students in Canadian medical schools. Canadian Medical Association Journal, 166(8), 1029-1035.

Entz, M., Slater, J., \& Desmarais, A.A. (2017). Student food insecurity at the University of Manitoba. Canadian Food Studies, 4(1), 139-159. doi: 10.15353/cfs-rcea.v4i1.204

Farahbakhsh, J., Hanbazaza, M., Ball, G.D., Farmer, A.P., Maximova, K., \& Willows, N.D. (2017). Food insecure student clients of a university-based food bank have compromised health, dietary intake and academic quality. Nutrition \& Dietetics, 74(1), 67-73. DOI: 10.1111/1747-0080.12307

Fink, A. (2013). How to conduct surveys: A step-by-step guide (5th ed.). Sage Publications.

Frank, L. (2018). "Hungry for an education": Prevalence and outcomes of food insecurity among students at a primarily undergraduate university in rural Nova Scotia. Canadian Journal of Higher Education, 48(2), 109-129. DOI: https://doi.org/10.47678/cjhe.v48i2.188112.

Health Canada. (2008). Canadian community health survey, cycle 2.2, nutrition (2004): Incomerelated household food security in Canada. https://www.canada.ca/content/dam/hcsc/migration/hc-sc/fn-an/alt_formats/hpfb-dgpsa/pdf/surveill/income_food_sec-sec_alimeng.pdf.

Hughes, R., Serebryanikova, I., Donaldson, K., \& Leveritt, M. (2011). Student food insecurity: The skeleton in the university closet. Nutrition \& Dietetics, 68(1), 27-32. https://doi.org/10.1111/j.1747-0080.2010.01496.x

Kirkpatrick, S.I., \& Tarasuk, V. (2008). Food insecurity is associated with nutrient inadequacies among Canadian adults and adolescents. The Journal of Nutrition, 138(3), 604-612. DOI: $10.1093 / \mathrm{jn} / 138.3 .604$

Maroto, M.E., Snelling, A., \& Linck, H. (2015). Food insecurity among community college students: Prevalence and association with grade point average. Community College Journal of Research and Practice 39(6), 515-526. DOI: 10.1080/10668926.2013.850758

Nurullah, A.S. (2010). Gender differences in distress: The mediating influence of life stressors and psychological resources. Asian Social Science, 6(5), 27. DOI:10.5539/ass.v6n5p27

Olauson, C., Engler-Stringer, R., Vatanparast, H., \& Hanoski, R. (2018). Student food insecurity: Examining barriers to higher education at the University of Saskatchewan. Journal of Hunger \& Environmental Nutrition, 13(1), 19-27. https://doi.org/10.1080/19320248.2017.1393365 
Patton-López, M.M., López-Cevallos, D.F., Cancel-Tirado, D.I., \& Vazquez, L. (2014). Prevalence and correlates of food insecurity among students attending a midsize rural university in Oregon. Journal of Nutrition Education and Behavior, 46(3), 209-214. DOI: 10.1016/j.jneb.2013.10.007

Silverthorn, D. (2016). Hungry for knowledge: Assessing the prevalence of student food insecurity on five Canadian campuses. https:/www.mealexchange.com/what-wedo/hungryforknowledgereport.

Smith-Carrier, T. (2020). Low funding for universities puts students at risk for cycles of poverty, especially in the wake of COVID-19. https://academicmatters.ca/low-funding-foruniversities-puts-students-at-risk-for-cycles-of-poverty-especially-in-the-wake-of-covid$19 /$

Statistics Canada. (2016). Women and education: Qualifications, skills and technology. https://www150.statcan.gc.ca/n1/pub/89-503-x/2015001/article/14640-eng.htm

Statistics Canada. (2020). Household food security by living arrangement. https://www150.statcan.gc.ca/t1/tbl1/en/tv.action?pid=1310038501\&pickMembers\%5B0 $\% 5 \mathrm{D}=1.1$

Tabachnick, B.G., \& Fidell, L.S. (2007). Using multivariate statistics (5th ed.). Allyn \& Bacon/Pearson Education.

Tarasuk, V., Mitchell, A., \& Dachner, N. (2014). Household food insecurity in Canada, 2012. Resource document. http://proof.utoronto.ca/wpcontent/uploads/2014/05/Household_Food_Insecurity_in_Canada-2012_ENG.pdf.

Tarasuk, V., Cheng, J., De Oliveira, C., Dachner, N., Gundersen, C., \& Kurdyak, P. (2015). Association between household food insecurity and annual health care costs. Canadian Medical Association Journal, 187(14), E429-E436. DOI: https://doi.org/10.1503/cmaj.150234

Tarasuk, V., Cheng, J., Gundersen, C., de Oliveira, C., \& Kurdyak, P. (2018). The relation between food insecurity and mental health care service utilization in Ontario. The Canadian Journal of Psychiatry, 63(8), 557-569. DOI: 10.1177/0706743717752879

Vozoris, N.T., \& Tarasuk, V.S. (2003). Household food insufficiency is associated with poorer health. The Journal of Nutrition, 133(1), 120-126. DOI: 10.1093/jn/133.1.120 